\title{
The Elderly Para- and Tetraplegic: Special Aspects of the Urological Care
}

\author{
G. Madersbacher, M.D. ${ }^{1}$ M. Oberwalder, M.D. ${ }^{2}$ \\ ${ }^{1}$ Consultant Urologist, Rehabilitation Center Bad Häring, A-6323 Bad Häring, \\ Tyrol, Austria and University Hospital A-6020 Innsbruck, Austria, ${ }^{2}$ Research \\ Assistant, University Hospital, Innsbruck A-6020, Austria
}

\section{Summary}

This paper evaluates the influence of age on bladder rehabilitation and the incidence of secondary changes in the urinary tract with long standing paraplegia and tetraplegia and its consequences for the elderly patient.

It demonstrates that age makes bladder rehabilitation in these patients more difficult, because of the decreased ability of the elderly to cope with the new situation, of pre-existing factors interfering with micturition like benign prostatic hyperplasia and cytocele and because of a delayed and at the end sometimes weak detrusor reflect activity. Long standing paraplegia is characterised by a high percentage of secondary structural changes in the lower urinary tract, especially at the bladder outlet. Once these changes are established the outflow resistance is further increased. These findings stress the need for early adequate management of the functional outflow obstruction based on careful urodynamic evaluation, before secondary structural changes occur. In this connection it is pointed out, that passive voiding by abdominal straining or by the Crede technique may cause unphysiologically high intravesical and intra-abdominal pressures and should then be replaced by intermittent catheterisation.

Key words: Neuropathic bladder in the elderly; Rehabilitation in the elderly; Long lasting paraplegia.

\section{Introduction}

In elderly paraplegic and tetraplegic patients two groups must be distinguished: one which suffers a spinal cord injury (SCI) as elderly and the other, which becomes old with a SCI. This study firstly evaluates the influence of age on the effectiveness of bladder rehabilitation, and secondly the incidence of structural changes in the urinary tract with long lasting paraplegia and tetraplegia. It is a retrospective analysis, based on the hospital charts of the Rehabilitation Center Bad Häring, Tyrol, Austria. 


\section{Patients and methods}

\section{Spinal cord injury after the age of 60-Group I}

This group contains 20 men and 6 women with a mean age of $69 \cdot 2$ years, who had a SCI after the age of 60 years. The mean duration of illness in this group is 3.6 years, 13 have complete and 13 incomplete lesions, 8 at the cervical level, the others at the thoracic level or below.

\section{Age over 60 and with a spinal cord injury for more than 20 years-Group II}

This group includes 22 men and 7 women; 15 have a complete and 14 incomplete lesions, the majority at the thoracic level or below. In order to study a homogeneous group only those with complete lesions were analysed. Of these 15 patients ( 12 men, 3 women) with complete lesions, 5 voided reflexly, 9 by abdominal straining, one is on intermittent catheterisation for 34 years, none were on permanent catheter drainage.

\section{Results}

Spinal cord injury after the age of 60-Group I

Type of bladder empyting. Only in 10 out of 20 men $(50 \%)$ balanced voiding by reflex, abdominal straining or spontaneously could be achieved, one patient catheterises himself, 9 required permanent transurethral or suprapubic bladder drainage, whereas 4 out of 6 women achieved balanced voiding and 2 are on intermittent self-catheterisation. These findings are also significant when studying the rate of urinary tract infections (UTI).

The incidence of urinary tract infections. Only 8 out of $26(31 \%)$ had a sterile urine, whereas the other $18(69 \%)$ were unsterile and had recurrent UTI. All patients on permanent bladder drainage had recurrent UTI, whereas all patients on intermittent (self)-catheterisation had sterile urine at their last assessment and this was the case for most of the previous period. Slight pyelonephritic changes, not present on the first IVP after the injury were found after a mean duration of illness of 3.6 years in 5 out of 52 kidneys, but in two of them the changes were only slight.

Operative procedures. Ten out of 20 men $(50 \%)$ have required 16 procedures; bladder calculi were only found in 3 patients, who had permanent bladder drainage, they required 6 lithotrypsies, and in 2 of them stones had to be removed more than once; 10 further procedures were undertaken to relieve outflow obstruction: a sphincterotomy together with a TUR of the prostate was necessary in 6 patients, and in 3 patients TUR of the prostate alone and in one patient a bladder neck incision only.

Age of 60 with a spinal cord injury for more than 20 years-Group II

Structural changes in the lower urinary tract. In $67 \%$ structural changes in the lower urinary tract were found: urethral diverticula at the peno-scrotal angle, reflux into the male adnexa, prostatic cavernes and calculi and, with increasing 
duration of illness more frequently, an increasing rigidity of the proximal posterior urethra and the bladder neck is seen in the voiding cystometrogram. These findings must be regarded as direct or indirect sequelae of dysfunction of the neurogenic bladder. All patients with a reflex bladder showed marked bladder trabeculation, but also 6 out of 9 patients who voided by straining and/or by the Crede procedure because of areflexia or hyporeflexia of the detrusor showed trabeculation and also sacculation.

Incidence of urinary tract infections. Only 5 out of 15 patients had a sterile urine when last seen, and among them was a patient who catheterised himself intermittently over a period of 34 years, whereas the other 10 patients had a positive urine culture with a history of recurrent urinary tract infection more than twice a year. There was a good correlation between recurrent UTI and secondary changes in the lower urinary tract.

Secondary changes in the upper urinary tract. In $37 \%$ of patients secondary changes were present; $6(20 \%)$ out of 29 kidneys showed pyelonephritic changes, a vesico uretero renal reflux was found into 5 renal units $(17 \%) ; 1$ patient, who had undergone a nephrectomy several years ago because of pyonephrosis and kidney stones, has renal insufficiency with a serum creatinine between 5-6 mg $\%$.

Operative procedures. In 8 patients (53\%) 17 operations had to be performed. In 6 to relieve bladder outflow obstruction mostly by sphincterotomy in combination with a TUR of the prostate or together with a bladder neck incision, in seven to correct a large urethral diverticulum; in two to remove stones from the upper urinary tract (kidney, urether); in one patient a kidney had to be removed because of pyronephrosis; and in one patient an antireflux procedure was performed. Sphincterotomy alone was not considered to be sufficient, and was therefore combined with either a TUR of the prostate, or a bladder neck incision, and this reflects the high incidence of secondary changes at the bladder outlet.

\section{Discussion}

There are two reasons why an increasing number of patients with SCI will reach the elderly age group, firstly the progress in the acute management has also improved the chances of an elderly patient to survive a SCI, and secondly, with adequate life time care more patients will become older with a SCI. Recently Eisenberg and Tierney (1985) stressed the changing demographic profile of the spinal cord injury population, with an increase in the numbers of older SCI persons requiring medical care. It is well known, that micturition problems increase with age, even in the general population. One must therefore assume that in the elderly patient with a SCI the neuropathic bladder is further complicated by age related factors. These facts stimulated us to review our elderly paraplegic and tetraplegic patients with regards to special aspects of their urological care.

This paper first considers in retrospect the effectiveness of 'bladder training' in a group of elderly paraplegic and tetraplegic patients who sustained the SCI after the age of 60 . In $54 \%$ of the male patients adequate bladder emptying could not be achieved: 9 out of 20 were on permanent catheter drainage when last seen. This percentage is much higher than in the younger age groups. 
Consequently there is a high rate of recurrent urinary tract infections and complications such as bladder calculi.

The reasons for instituting permanent urinary drainage were a poor general condition: cerebral sclerosis in 2; nursing problems such as difficulties with catheterisation or to manage urinary incontinence in $4 ; 2$ patients refused an operation at the bladder outlet as well as refusing intermittent catheterisation or any form of appliance, and 1 patient who had already had two previous unsuccessful operations elsewhere to relieve bladder outflow obstruction declined a third operative procedure.

The following facts account for the difficulties in bladder rehabilitation in the elderly paraplegic or tetraplegic, (1) the decreased ability of the elderly to cope with the new situation, (2) pre-existing factors interfering with micturition, such as benign prostate hyperplasia and cystocele, (3) a delayed and subsequently a weak detrusor reflex activity and (4) the erroneous belief, of both doctors and nurses, that continuous cathether drainage is a safe and comfortable way to solve the problem. There are therefore a variety of reasons, some of which cannot be reversed, accounting for the difficulties. On the patient's side we often find that the motivation for rehabilitation maybe lacking in the elderly. However it should be stressed that in this older age group intermittent catheterisation in general is definitely preferable to any kind of permanent bladder drainage.

The second part of this paper deals with a group of paraplegic and tetraplegic patients over 60 years of age with a spinal cord injury that occurred 20 or more years ago. It focuses on a rather neglected area of urological practice. We are in agreement with England and Low (1985) stating, that there is now an extensive literature on the acute and early management of the neuropathic bladder in paraplegia but very few reports are available on the long-term urological care of these patients (O'Flynn, 1978; Golji, 1980). This group of patients with longlasting spinal cord injury is characterised by a high percentage of secondary morphologic changes in the lower urinary tract due to a persisting functional outflow obstruction which is almost unavoidable in reflex voiding as well as with voiding by abdominal straining or the Crede procedure. Chronic prostatitis, caused by overpressure, mechanical and chemical irritation and lastly by true infection is one of these consequences and is therefore common in the spiral paralysed, the frequency of which is steadily rising with the duration of the paralysis (Madersbacher, 1977). Both factors, the dysfunction of micturition itself as well as these secondary structural alterations may change the physical properties-shape, elasticity and consistency of the posterior urethra and of the bladder neck, thus making the flow conditions even worse, as has also been demonstrated experimentally (Madersbacher et al., 1980). These facts stress the need for early adequate management before these secondary structural changes occur. Detrusor striated sphincter dyssynergia is well accepted nowadays as a common finding in upper motor neuron lesions, moreover the indications for and the different ways to manage it are known. But passive voiding by abdominal straining and/or the Crede technique are still widely regarded as the method of choice for bladder emptying in patients with lower motor neuron lesions. The unphysiologically high intravesical pressures which are usually necessary to overcome the functional obstruction which is present during this form of voiding 
at the level of the membranous urethra even with a totally flaccid paralysed pelvic floor are often neglected. Sacculation and trabeculation are consequences which were found in 6 out of 9 patients in this series, who voided by straining and/or by using the Crede manoeuvre for 20 years and more because of areflexia or hyporeflexia of the detrusor. We feel that this rather unphysiological way of bladder emptying should be replaced by intermittent (self)-catheterisation at least in patients, in whom cystometry demonstrates unphysiological high intravesical voiding pressures. The status of the lower urinary tract and its consequences for the upper urinary tract in this group of patients with long lasting paraplegia clearly demonstrates the importance of a careful urodynamic investigation as has already been stated by Rossier (1979) in order to detect patients who are at risk in time, and to decide on the proper therapy for the individual patient. Due to functional and structural changes the neuropathic bladder is not a static, but is a dynamic phenomenon. Therefore the situation may change in time and regular follow-up studies are necessary to prevent possible functional and structural deterioration of the urinary tract in patients with long lasting spinal cord injury.

\section{Conclusions}

From our findings in two groups of elderly tetraplegic and paraplegic patients we conclude for group I that age makes bladder rehabilitation in patients with spinal cord injury more difficult for various reasons: because of the decreased ability of the elderly paraplegic to cope with the new situation, of pre-existing factors interfering with micturition like BPH and cystocele, because of a delayed and at the end weak detrusor reflex activity and, last but not least, because of the still existing erroneous belief of those looking after these elderly patients that continuous catheter drainage is a safe and comfortable way to solve this problem. Group II demonstrates that patients with long standing paraplegia or tetraplegia show a high percentage of secondary structural abnormalities in the lower urinary tract due to persisting outflow obstruction, which in turn make flow conditions even worse demanding for a further increase of an already unphysiological high intravesical voiding pressure. This stresses the need for early adequate management based on careful urodynamic investigations before secondary structural abnormalities occur. In this regard it is also pointed out that voiding by abdominal straining and/or using the Crede procedure may cause unphysiological high voiding pressures and may therefore be a dangerous way of bladder emptying; thus it should be replaced by intermittent bladder catheterisation.

\section{References}

Eisenberg MG, Tierney DO 1985 Changing demographic profile of the spinal cord injury population: Implications for health care support systems. Paraplegia 23: 335-343.

England EJ Low AI 1985 Lifetime Care of the Paraplegic Patient by Sir George M. Bedbrook, Churchill Livingstone, Edinburgh, London, Melbourne and New York, pp. 94-105: Long term management and prevention of urinary tract disease.

GoLjI G 1980 Urethral sphincterotomy for chronic spinal cord inury. Fournal of Urology 123: 204-207.

MADERSBACHER H 1977 The neuropathic urethra: urethrogram and pathophysiologic aspects. European Urology 3: 321-332. 
MAdersbaCher H, Marberger H, Holl G 1980 Hydrodynamic aspects of bladder-outlet obstruction: consequences of functional micturition disorders. Paraplegia 18: 307-313. O'FLYNN JD 1978 Early and late management of the neuropathic bladder in spinal cord injury patients. Fournal of Urology 120: 726-728.

Rossier AB, FAM BA 1979 From Intermittent catheterization to catheter freedom via urodynamics: a tribute to Sir Ludwig Guttmann. Paraplegia 17: 73-85. 